Revista peruana de biología 26(1): 049 - 062 (2019) doi: http://dx.doi.org/10.15381/rpb.v26i1.15909 ISSN-L 1561-0837; elSSN: 1727-9933

Universidad Nacional Mayor de San Marcos

\section{Microestructura cuticular y medular del pelo de guardia de mamíferos pequeños terrestres en la región de Arequipa, Perú}

\section{TRABAJOS ORIGINALES}

$\begin{array}{ll}\text { Presentado: } & 06 / 08 / 2018 \\ \text { Aceptado: } & 24 / 01 / 2019 \\ \text { Publicado online: } & 30 / 03 / 2019\end{array}$

Correspondencia:

*Autor para correspondencia

1 Universidad Nacional de San Agustín de Arequipa, Museo de Historia Natural (MUSA), Colección científica. Av. Alcides Carrión s/n. Arequipa, Perú. 2 Universidad Nacional de San Agustín de Arequipa, Facultad de Ciencias Biológicas, Departamento Académico de Biología, Sección de Zoología. Av. Alcides Carrión s/n. Arequipa, Perú.

Email YKM: yasmymedina@gmail.com Email CEM: cmedinap1234@yahoo.com Email EL: elopezt@yahoo.es

Citación:

Medina Y.K., C.E. Medina, E. López. 2019. Microestructura cuticular y medular del pelo de guardia de mamíferos pequeños terrestres en la región de Arequipa, Perú. Revista peruana de biología 26(1): 049 - 062 (Febrero 2019). doi: http://dx.doi. org/10.15381/rpb.v26i1.15909

Palabras clave: Cutícula; mamífero; marsupial; médula; roedor.

Keywords: Cuticle; mammal; marsupial; marrow; rodent.

\section{Cuticular and medullar microstructure of the guard hair of small land mammals in the region of Arequipa, Peru}

\author{
Yasmy K. Medina*1,2, César E. Medina ${ }^{1,2}$, Evaristo López ${ }^{1,2}$ \\ 1 Universidad Nacional de San Agustín de Arequipa, Museo de Historia Natural \\ (MUSA), Perú. \\ 2 Universidad Nacional de San Agustín de Arequipa, Facultad de Ciencias Biológicas, \\ Perú.
}

\begin{abstract}
Resumen
Actualmente, el estudio de pelos de guardia está siendo utilizado en diversas áreas de las ciencias básicas y aplicadas (taxonomía, ecología, genética, paleontología, criminalística, entre otras), debido a su resistencia a factores físicos, químicos, mecánicos y biológicos, conservando su estructura cuticular y medular. Dichas estructuras forman patrones que permiten la identificación de especímenes a nivel de géneros y familias. Sin embargo, a pesar de la importancia, no hay estudios sobre este tema en Perú. Por ello, con el fin de llenar este vacío de información, se examinaron muestras de 30 especies de mamíferos de la región de Arequipa, correspondientes a los órdenes Didelphimorphia y Rodentia, donde encontramos 5 patrones medulares y 8 cuticulares. Los marsupiales didélfidos presentan un patrón medular uniseriado escaleriforme con un patrón cuticular foliáceo. En los roedores, los cricétidos tienen un patrón medular multiseriado alveolar y un patrón cuticular foliáceo, los chinchílidos un patrón medular reticular con patrón cuticular pétalo diamante tipo $D$, los cávidos un patrón medular reticular con patrón cuticular ondeado transversal, los abrocómidos un patrón medular listrado con patrón cuticular ondeado oblicuo simple, y los múridos un patrón medular reticular y alveolar con patrón cuticular pétalo diamante (tipo B y C) y foliáceo. Presentamos por primera vez la descripción detallada de la cutícula y médula de los pelos de guardia de 24 especies de roedores y 2 marsupiales.
\end{abstract}

\section{Abstract}

Currently, the study of guard hairs is used in various areas of basic and applied sciences (taxonomy, ecology, genetics, paleontology, criminology, among others), due to its resistance to physical, chemical, mechanical and biological factors, conserving its cuticular and medullar structure. These structures form patterns that allow the identification of specimens at the level of genera and families. However, despite the importance, there are no studies on this subject in Peru. Therefore, samples from 30 mammalian species of Didelphimorphia and Rodentia were examined to fill this information gap. Among the species studied, we differentiated 5 medullary and 8 cuticular patterns. The didelphid marsupials have a medial uniseriate scaleriform pattern with a foliaceous cuticular pattern; the cricetid rodents have an alveolar multiseriate medullary pattern and a foliate cuticular pattern, the chinchillids a reticular medullary pattern with a cuticular pattern of diamond petal type $D$, the caviids a reticular medullary pattern with a transverse cuticular pattern, the abrocomids a medullary pattern listrade with simple oblique wave cuticular pattern, and the murids a reticular and alveolar medullary pattern with cuticular pattern petal diamond (type B and C) or foliaceous. We present for the first time the detailed description of the cuticle and marrow of the guard hairs of 24 species of rodents and 2 marsupials. 


\section{Introducción}

El pelo como medio de identificación de los mamíferos ha sido ampliamente estudiado a nivel mundial (Hausman 1920, Benedict 1957, Keller 1980, De Marinis \& Agnelli 1993, Albayrak \& Coban 1997, Vázquez et al. 2000, Martin et al. 2001, Ibarra \& Sanchez-Cordero 2004, Quadros \& Monteiro-Filho 2006a, Pech-Canché et al. 2009, Gómez \& Cassini 2010, Sarkar et al. 2013, Felix et al. 2014, Kumar et al. 2014); sin embargo, en Sudamérica sólo se conocen estudios en Argentina, Guatemala y Brasil (Fernández \& Rossi 1998, Martin et al. 2009, Juárez et al. 2007, Silveira et al. 2013, Gatto-Almeida et al. 2016).

A pesar de que, en la región Arequipa se tiene registro de 72 especies de mamíferos silvestres y 13 especies introducidas, la información sobre la morfología del pelo de dichas especies es inexistente. De acuerdo con lo anterior, el objetivo del presente trabajo es describir las características de la microestructura cuticular y medular de las especies de los órdenes Didelphimorphia y Rodentia que habitan en la región Arequipa, de modo que, este estudio podría ser una base para avanzar en las actividades de investigación pertinentes en esta área.

\section{Material y métodos}

Para la descripción de pelos y patrones se siguieron los trabajos de Hausman (1920), Benedict (1957), Day (1966), Perrin y Campbell (1980), Arita y Aranda (1987), Teerink (1991), Robertson (1999), Quadros y MonteiroFilho (2006a) y Debelica y Thies (2009) (Fig. 1).

Se examinaron 30 especies de mamíferos pequeños correspondientes a los órdenes Didelphimorphia (2 especies silvestres) y Rodentia (25 silvestres y 3 introducidas). Se siguieron los trabajos de Zeballos et al. (2001); Palma et al. (2014); Zeballos et al. (2014); Patton et al. (2015) y do Prado y Percequillo (2018) para el arreglo sistemático (Tabla 1).

Los pelos de guardia fueron obtenidos de especímenes depositados en la colección científica de mamíferos del Museo de Historia Natural (MUSA ) (Apéndice I). La colecta de pelos de guardia se realizó de la zona interescapular de tres individuos adultos por especie, escogidos al azar, siguiendo la metodología de Arita y Aranda (1987), Monroy-Vilches et al. (2005) y Quadros y Monteiro-Filho (2006b). Se observaron 10 pelos de guardia por espécimen.

Tabla 1. Listado de los mamíferos pequeños terrestres examinados en este trabajo

\begin{tabular}{|c|c|c|}
\hline Orden & Familia & Especie \\
\hline \multicolumn{3}{|l|}{ Mamíferos silvestres } \\
\hline \multirow{2}{*}{ Didelphimorphia } & \multirow{2}{*}{ Didelphidae } & Thylamys pallidior (Thomas, 1902) \\
\hline & & Thylamys sp. \\
\hline \multirow{25}{*}{ Rodentia } & \multirow{21}{*}{ Cricetidae } & Abrothrix andinus Philippi, 1858 \\
\hline & & Abrothrix jelskii Thomas, 1894 \\
\hline & & Akodon subfuscus Osgood, 1944 \\
\hline & & Akodon albiventer Thomas, 1897 \\
\hline & & Auliscomys boliviensis (Waterhouse, 1846) \\
\hline & & Auliscomys pictus (Thomas, 1884) \\
\hline & & Auliscomys sublimis (Thomas, 1900) \\
\hline & & Calomys lepidus (Thomas, 1884) \\
\hline & & Calomys miurus (Thomas, 1926) \\
\hline & & Calomys chinchilico Zeballos, Palma, Marquet y Ceballos, 2014 \\
\hline & & Calomys achaku Zeballos, Palma, Marquet y Ceballos, 2014 \\
\hline & & Chinchillula sahamae Thomas, 1898 \\
\hline & & Necromys amoenus (Thomas, 1900) \\
\hline & & Neotomys ebriosus Thomas, 1894 \\
\hline & & Oligoryzomys andinus (Osgood, 1914) \\
\hline & & Oligoryzomys arenalis Thomas, 1913 \\
\hline & & Aegialomys ica (Osgood, 1944) \\
\hline & & Phyllotis amicus Thomas, 1900 \\
\hline & & Phyllotis limatus Thomas, 1912 \\
\hline & & Phyllotis magister Thomas, 1912 \\
\hline & & Phyllotis xanthopygus (Waterhouse, 1837) \\
\hline & Chinchillidae & Lagidium viscacia (G.I. Molina, 1782) \\
\hline & \multirow{2}{*}{ Caviidae } & Cavia porcellus (Linnaeus, 1758) \\
\hline & & Cavia tschudii Fitzinger, 1857 \\
\hline & Abrocomidae & Abrocoma cinerea Thomas, 1919 \\
\hline \multicolumn{3}{|c|}{ Mamíferos introducidos } \\
\hline \multirow{3}{*}{ Rodentia } & \multirow{3}{*}{ Muridae } & Rattus rattus (Linnaeus, 1758) \\
\hline & & Rattus norvegicus (Berkenhout, 1769) \\
\hline & & Mus musculus Linnaeus, 1758 \\
\hline
\end{tabular}




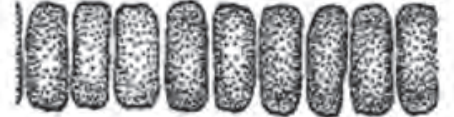

1
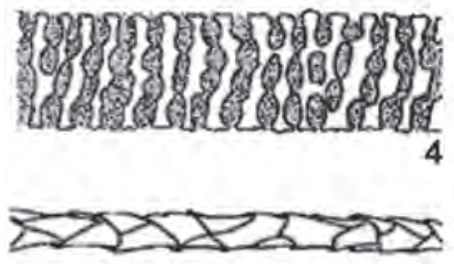

7A

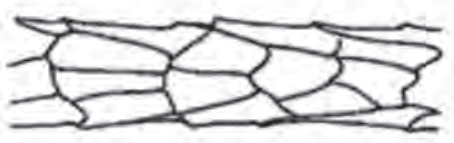

7D

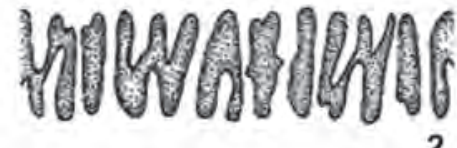

2
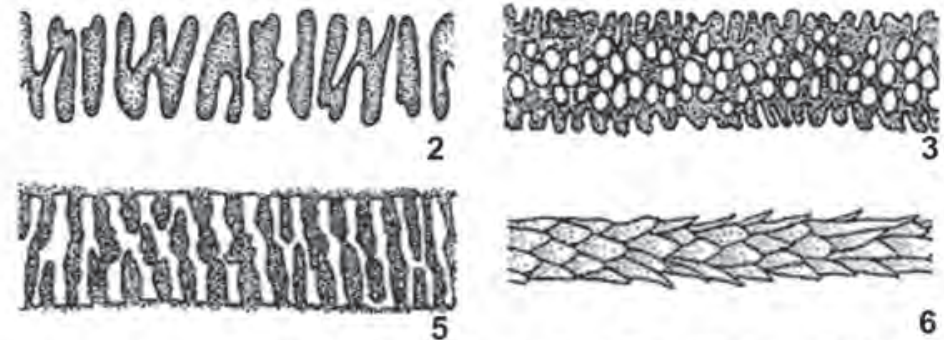

5

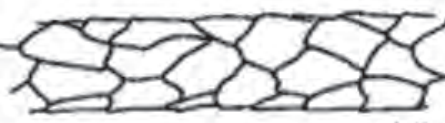

7B

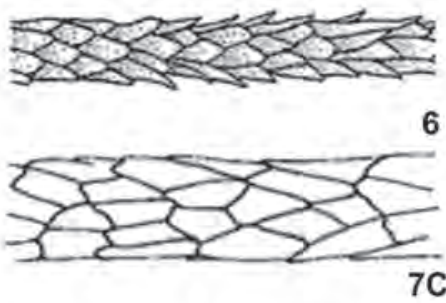

7C

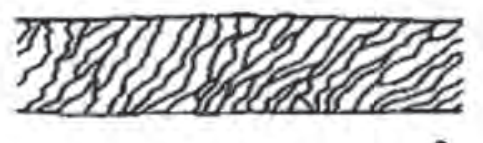

8

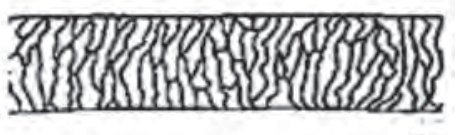

9

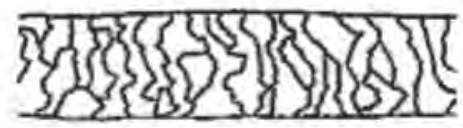

10

Figura 1: Representación esquemática de patrones medulares y cuticulares reconocidos en este estudio, tomados de Quadros y Monteiro-Filho (2006b). Médula: Uniseriada escaleriforme (1), Uniseriada literoides (2), Multiseriada reticular (3), Multiseriada alveolar (4), Multiseriada listrada (5). Cutícula: Patrón foliáceo estrecho (6), Patrón pétalo diamante "tipo A" (7A), Patrón pétalo diamante "tipo B" (7B), Patrón pétalo diamante "tipo C" (7C), Patrón pétalo diamante "tipo D" (7D) (Day 1966, Teerink 1991), Ondeado oblicuo simple (8), Ondeado transversal crenado (9), Ondeado transversal liso (10).

La limpieza se realizó con solución jabonosa y luego con alcohol según Fasola et al. (2005) y Kshirsagar et al. (2009). Las láminas de impresión cuticular se obtuvieron con ayuda de barniz de uñas transparente y láminas portaobjetos (Brunner \& Coman 1974 y Juárez et al. 2007), generándose un negativo de las escamas de la superficie de los pelos. En cambio, las láminas medulares fueron obtenidas fijándose los pelos con ayuda de Bálsamo de Canadá, previamente decolorados con peróxido de hidrogeno, sobre las láminas portaobjetos (Gurini 1985, Arita \& Aranda 1987). Las láminas permanentes fueron depositadas en el Museo de Historia Natural de la Universidad Nacional de San Agustín de Arequipa (MUSA). Las láminas fueron revisadas con un microscopio óptico a 100 aumentos, observándose por cada muestra 4 porciones del pelo: la porción proximal, la parte media de la asta del pelo para caracterizar la cutícula, el escudo en la porción más ancha para caracterizar la médula (dado que en esta zona las escamas de la cutícula y las células de la médula son más uniformes y maduras) y la porción distal (Teerink 1991, Debelica \& Thies 2009) (Fig. 2).

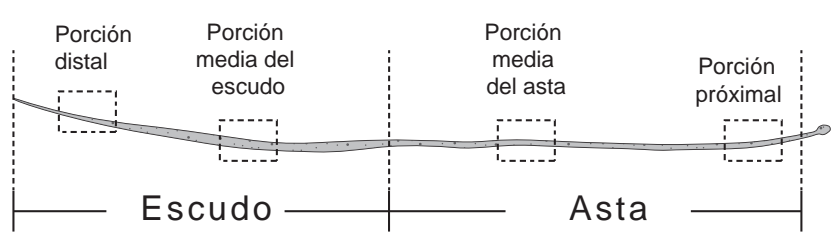

Figura 2: Representación esquemática de las cuatro porciones observadas en el pelo de guardia tipo arista fusiforme. (Teerink 1991, Quadros 2002, Debelica \& Thies 2009).
Se tomaron también las siguientes medidas macroscópicas y microscópicas: longitud del pelo (LP), ancho del escudo, ancho de la médula, ancho de la corteza, índice de medulación (relación entre el ancho de médula y ancho de escudo), índice médula/corteza, largo de escama, ancho de escama y el índice de escama (relación entre el largo de escama y ancho de escama). Cada medida fue obtenida del promedio de 30 mediciones por especie (Cavia et al. 2008, Anwar et al. 2012).

\section{Resultados}

En total se analizaron 89 especímenes, correspondientes a 27 especies silvestres y tres introducidas, agrupados en 16 géneros, 6 familias y 2 órdenes ( 2 marsupiales y 28 roedores) (Tabla 1 ).

Al examinar externamente los pelos de guardia, se observó que todos los individuos analizados presentan pelo de guardia de tipo arista (pelos con el crecimiento definitivo que tienen la parte distal amplia y reforzada, con una base más pequeña y más débil) y con forma de huso (fusiformes).

En cuanto a los datos cualitativos, en todas las especies analizadas la médula está presente; en la mayoría de las especies analizadas la parte proximal del pelo presenta un patrón medular multiseriado (Lagidium viscacia) y uniseriado literoides (Rattus rattus, Cavia porcellus y C. tschudii) o uniseriado escaleriforme (resto de especies) y en la cutícula las escamas tienen forma de pétalo diamante (Fig. 1). En la parte distal del pelo la médula se va deformando hasta desaparecer, mientras que las es- 
camas de la cutícula se van apiñando y el borde externo sufre un desgaste que provoca la formación de un patrón ondeado transversal y a veces ornamentado (crenado).

En la región del escudo se hallaron 4 patrones medulares y en la región de la asta se hallaron 6 patrones cuticulares (Fig. 1), de los cuales el patrón medular escaleriforme sólo se presenta en el orden Didelphimorphia a lo largo de todo el pelo; mientras que, los patrones medulares son de distintas formas en el orden Rodentia (Tablas 2 y 3). A continuación, se presentan los patrones observados en el escudo (médula) y asta (cutícula) de las familias estudiadas:

Didelphidae.- Los representantes de esta familia se caracterizaron por la presencia de una médula yuxtapuesta (paredes de células adyacentes en contacto) uniseriada, de bordes celulares íntegros y células medulares escaleriformes en todo el eje del pelo, con un patrón cuticular imbricado foliáceo estrecho, de orientación oblicua simple y con bordes continuos (Fig. 3: C y D). No se encontraron diferencias cualitativas ni cuantitativas entre las dos especies de dicha familia (Thylamys sp. y Thylamys pallidior)

Chinchillidae.- El representante de esta familia, $L a$ gidium viscacia, se caracterizó por presentar una médula anastomosada (fusión entre células adyacentes que definen diferentes formas y tamaños), de bordes celulares íntegros y forma de células reticulada, con una cutícula imbricada de forma pétalo diamante "tipo D", escamas estrechas y lisas, de orientación simple y bordes continuos (Fig. 4: C y D).

Caviidae.- Los representantes de esta familia se caracterizaron por presentar un patrón medular anastomosado reticular con bordes crenados, con una cutícula pavimentada, ondeada transversal, con escamas anchas y de bordes continuos (Fig. 5: C y D). No se encontraron diferencias cualitativas ni cuantitativas entre las dos especies de esta familia (Cavia tschudii y C. porcellus).

Abrocomidae.- El representante de esta familia, Abrocoma cinerea, se caracterizó por presentar un patrón medular anastomosado listrado con bordes íntegros, con un patrón cuticular ondeado oblicuo, de escamas anchas, crenadas y de bordes continuos (Fig. 6: C y D).

Cricetidae.- Los representantes de esta familia se caracterizaron por presentar un patrón medular anastomosado alveolar con bordes íntegros y una cutícula imbricada foliácea oblicua simple y de escamas estrechas sin ornamentaciones y con bordes continuos (Fig. 7: C y D). No se encontraron diferencias cualitativas ni cuan-

Tabla 2. Matriz de patrones medulares de los mamíferos pequeños terrestres en la región Arequipa.

\begin{tabular}{|c|c|c|c|c|c|}
\hline Especie / Características & Continuidad & Número de filas & Disposición de células & Ornamentación de bordes & Forma de célula \\
\hline \multicolumn{6}{|l|}{ Mamíferos silvestres } \\
\hline Thylamys pallidior & Discontinua & Uniseriada & Yuxtapuesta & Integra & Escaleriforme \\
\hline Thylamys sp. & Discontinua & Uniseriada & Yuxtapuesta & Integra & Escaleriforme \\
\hline Abrothrix andinus & Continua & Multiseriada & Anastomosada & Integra & Alveolar \\
\hline Abrothrix jelskii & Continua & Multiseriada & Anastomosada & Integra & Alveolar \\
\hline Akodon subfuscus & Continua & Multiseriada & Anastomosada & Integra & Alveolar \\
\hline Akodon albiventer & Continua & Multiseriada & Anastomosada & Integra & Alveolar \\
\hline Auliscomys boliviensis & Continua & Multiseriada & Anastomosada & Integra & Alveolar \\
\hline Auliscomys pictus & Continua & Multiseriada & Anastomosada & Integra & Alveolar \\
\hline Auliscomys sublimis & Continua & Multiseriada & Anastomosada & Integra & Alveolar \\
\hline Calomys chinchilico & Continua & Multiseriada & Anastomosada & Integra & Alveolar \\
\hline Calomys achaku & Continua & Multiseriada & Anastomosada & Integra & Alveolar \\
\hline Calomys lepidus & Continua & Multiseriada & Anastomosada & Integra & Alveolar \\
\hline Calomys miurus & Continua & Multiseriada & Anastomosada & Integra & Alveolar \\
\hline Chinchillula sahamae & Continua & Multiseriada & Anastomosada & Integra & Alveolar \\
\hline Necromys amoenus & Continua & Multiseriada & Anastomosada & Integra & Alveolar \\
\hline Neotomys ebriosus & Continua & Multiseriada & Anastomosada & Integra & Alveolar \\
\hline Oligoryzomys andinus & Continua & Multiseriada & Anastomosada & Integra & Alveolar \\
\hline Oligoryzomys arenalis & Continua & Multiseriada & Anastomosada & Integra & Alveolar \\
\hline Aegialomys ica & Continua & Multiseriada & Anastomosada & Integra & Alveolar \\
\hline Phyllotis amicus & Continua & Multiseriada & Anastomosada & Integra & Alveolar \\
\hline Phyllotis limatus & Continua & Multiseriada & Anastomosada & Integra & Alveolar \\
\hline Phyllotis magister & Continua & Multiseriada & Anastomosada & Integra & Alveolar \\
\hline Phyllotis xanthopygus & Continua & Multiseriada & Anastomosada & Integra & Alveolar \\
\hline Lagidium peruanun & Continua & Multiseriada & Anastomosada & Integra & Reticular \\
\hline Cavia porcellus & Continua & Multiseriada & Anastomosada & Crenada & Reticular \\
\hline Cavia tschudii & Continua & Multiseriada & Anastomosada & Crenada & Reticular \\
\hline Abrocoma cinerea & Continua & Multiseriada & Anastomosada & Integra & Listrada \\
\hline \multicolumn{6}{|l|}{ Mamíferos introducidos } \\
\hline Rattus rattus & Continua & Multiseriada & Anastomosada & Integra & Reticular \\
\hline Rattus norvegicus & Continua & Multiseriada & Anastomosada & Integra & Alveolar \\
\hline Mus musculus & Continua & Multiseriada & Anastomosada & Integra & Alveolar \\
\hline
\end{tabular}


Tabla 3. Matriz de característica externa y patrones cuticulares de los mamíferos pequeños terrestres en la región Arequipa. Código de bandas: O: Oscuro y C: Claro.

\begin{tabular}{|c|c|c|c|c|c|c|c|}
\hline \multirow[b]{2}{*}{ Especie / Características } & \multirow{2}{*}{$\begin{array}{l}\text { Externa } \\
\text { Código } \\
\text { Bandas }\end{array}$} & \multicolumn{6}{|c|}{ Patrones Cuticulares } \\
\hline & & $\begin{array}{c}\text { Superposición } \\
\text { de bordes }\end{array}$ & $\begin{array}{c}\text { Orientación } \\
\text { escamas }\end{array}$ & $\begin{array}{l}\text { Dimensión } \\
\text { escamas }\end{array}$ & Forma escama & $\begin{array}{l}\text { Orientación } \\
\text { borde escama }\end{array}$ & $\begin{array}{c}\text { Forma de borde de } \\
\text { escama }\end{array}$ \\
\hline \multicolumn{8}{|l|}{ Mamíferos silvestres } \\
\hline Thylamys pallidior & $\mathrm{O}-\mathrm{C}$ & Imbricado & Oblicuo & Estrecha & Foliácea & Lisa & Acuminado \\
\hline Thylamys sp. & $\mathrm{O}-\mathrm{C}$ & Imbricado & Oblicuo & Estrecha & Foliácea & Lisa & Acuminado \\
\hline Abrothrix andinus & $\mathrm{O}-\mathrm{C}$ & Imbricado & Oblicuo & Estrecha & Foliácea & Lisa & Acuminado \\
\hline Abrothrix jelskii & $\mathrm{O}-\mathrm{C}$ & Imbricado & Oblicuo & Estrecha & Foliácea & Lisa & Acuminado \\
\hline Akodon subfuscus & $\mathrm{O}-\mathrm{C}$ & Imbricado & Oblicuo & Estrecha & Foliácea & Lisa & Acuminado \\
\hline Akodon albiventer & $\mathrm{O}-\mathrm{C}$ & Imbricado & Oblicuo & Estrecha & Foliácea & Lisa & Acuminado \\
\hline Auliscomys boliviensis & $\mathrm{O}-\mathrm{C}-\mathrm{O}$ & Imbricado & Oblicuo & Estrecha & Foliácea & Lisa & Acuminado \\
\hline Auliscomys pictus & $\mathrm{O}-\mathrm{C}$ & Imbricado & Oblicuo & Estrecha & Foliácea & Lisa & Acuminado \\
\hline Auliscomys sublimis & $\mathrm{O}-\mathrm{C}-\mathrm{O}$ & Imbricado & Oblicuo & Estrecha & Foliácea & Lisa & Acuminado \\
\hline Calomys chinchilico & $\mathrm{O}-\mathrm{C}$ & Imbricado & Oblicuo & Estrecha & Foliácea & Lisa & Acuminado \\
\hline Calomys achaku & $\mathrm{O}-\mathrm{C}$ & Imbricado & Oblicuo & Estrecha & Foliácea & Lisa & Acuminado \\
\hline Calomys lepidus & $\mathrm{O}-\mathrm{C}$ & Imbricado & Oblicuo & Estrecha & Foliácea & Lisa & Acuminado \\
\hline Calomys miurus & $\mathrm{O}-\mathrm{C}-\mathrm{O}$ & Imbricado & Oblicuo & Estrecha & Foliácea & Lisa & Acuminado \\
\hline Chinchillula sahamae & $\mathrm{O}-\mathrm{C}-\mathrm{O}$ & Imbricado & Oblicuo & Estrecha & Foliácea & Lisa & Acuminado \\
\hline Necromys amoenus & $\mathrm{O}-\mathrm{C}$ & Imbricado & Oblicuo & Estrecha & Foliácea & Lisa & Acuminado \\
\hline Neotomys ebriosus & $\mathrm{O}-\mathrm{C}-\mathrm{O}$ & Imbricado & Oblicuo & Estrecha & Foliácea & Lisa & Acuminado \\
\hline Oligoryzomys andinus & $\mathrm{O}-\mathrm{C}-\mathrm{O}$ & Imbricado & Oblicuo & Estrecha & Foliácea & Lisa & Acuminado \\
\hline Oligoryzomys arenalis & $\mathrm{O}-\mathrm{C}$ & Imbricado & Oblicuo & Estrecha & Foliácea & Lisa & Acuminado \\
\hline Aegialomys ica & $\mathrm{O}-\mathrm{C}$ & Imbricado & Oblicuo & Estrecha & Foliácea & Lisa & Acuminado \\
\hline Phyllotis amicus & $\mathrm{O}-\mathrm{C}-\mathrm{O}$ & Imbricado & Oblicuo & Estrecha & Foliácea & Lisa & Acuminado \\
\hline Phyllotis limatus & $\mathrm{O}-\mathrm{C}-\mathrm{C}$ & Imbricado & Oblicuo & Estrecha & Foliácea & Lisa & Acuminado \\
\hline Phyllotis magister & $\mathrm{O}-\mathrm{C}-\mathrm{C}$ & Imbricado & Oblicuo & Estrecha & Foliácea & Lisa & Acuminado \\
\hline Phyllotis xanthopygus & $\mathrm{O}-\mathrm{C}$ & Imbricado & Oblicuo & Estrecha & Foliácea & Lisa & Acuminado \\
\hline Lagidium peruanun & $\mathrm{C}-\mathrm{O}$ & Imbricado & Oblicuo & Estrecha & Pétalo diamante “D” & Lisa & Alargado \\
\hline Cavia porcellus & $\mathrm{C}-\mathrm{C}$ & Pavimentado & Transversal & Ancha & Ondeada & Lisa & --- \\
\hline Cavia tschudii & $\mathrm{C}-\mathrm{O}-\mathrm{C}$ & Pavimentado & Transversal & Ancha & Ondeada & Lisa & --- \\
\hline Abrocoma cinerea & $\mathrm{O}-\mathrm{C}$ & Pavimentado & Oblicuo & Ancha & Ondeada & Adornada & Crenado \\
\hline \multicolumn{8}{|l|}{ Mamíferos introducidos } \\
\hline Rattus rattus & $\mathrm{O}-\mathrm{C}$ & Imbricado & Irregular & Intermedia & Pétalo diamante "C" & Lisa & Acuminado \\
\hline Rattus norvegicus & - & Imbricado & Oblicuo & Estrecha & Foliácea & Lisa & Acuminado \\
\hline Mus musculus & - & Imbricado & Transversal & Estrecha & Pétalo diamante "B" & Lisa & Acuminado \\
\hline
\end{tabular}

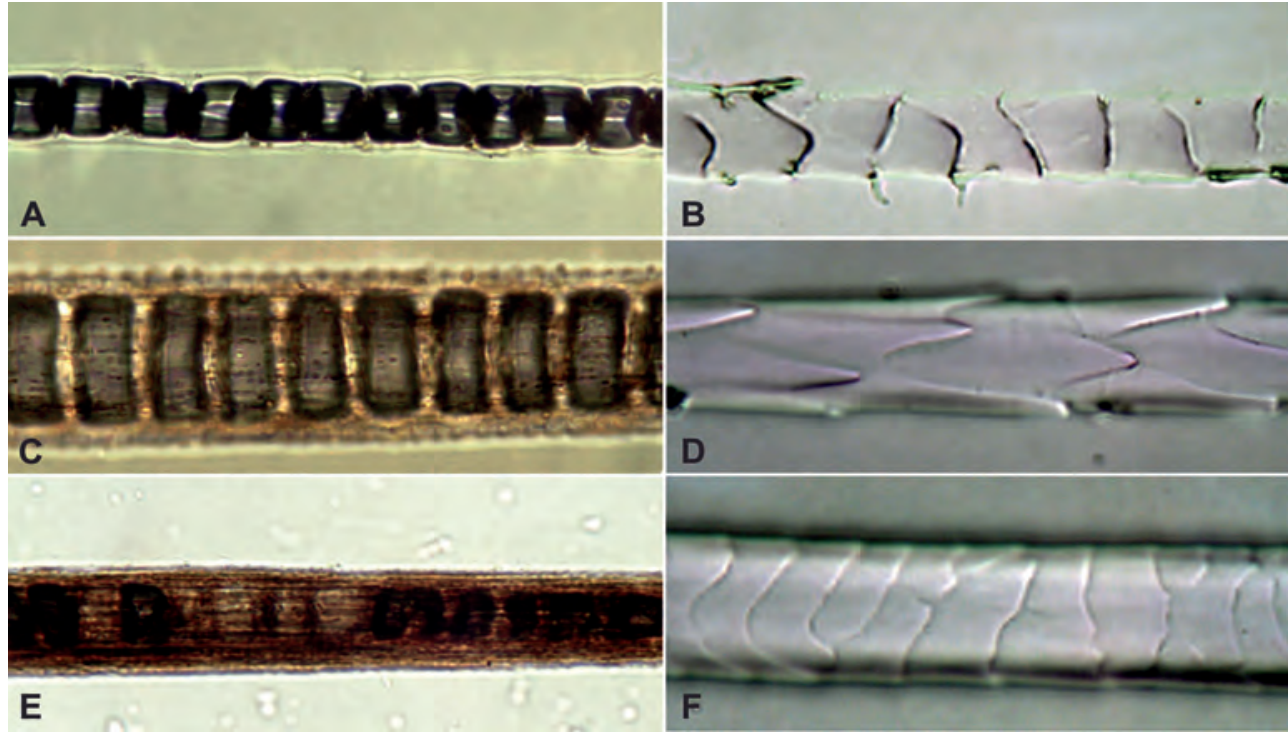

Figura 3: Patrones medulares y cuticulares de Thylamys spp.: Fotos de la izquierda Thylamys sp. (MUSA 11553) y fotos de la derecha Thylamys pallidior (MUSA 14400). Porción proximal: Médula escaleriforme uniseriada (A) y Cutícula pétalo diamante "tipo A" (B). Escudo: Médula escaleriforme uniseriada (C). Asta: Cutícula foliácea estrecha (D). Porción distal: Médula en deformación (E) y Escamas ondeadas transversales y lisas (F). 

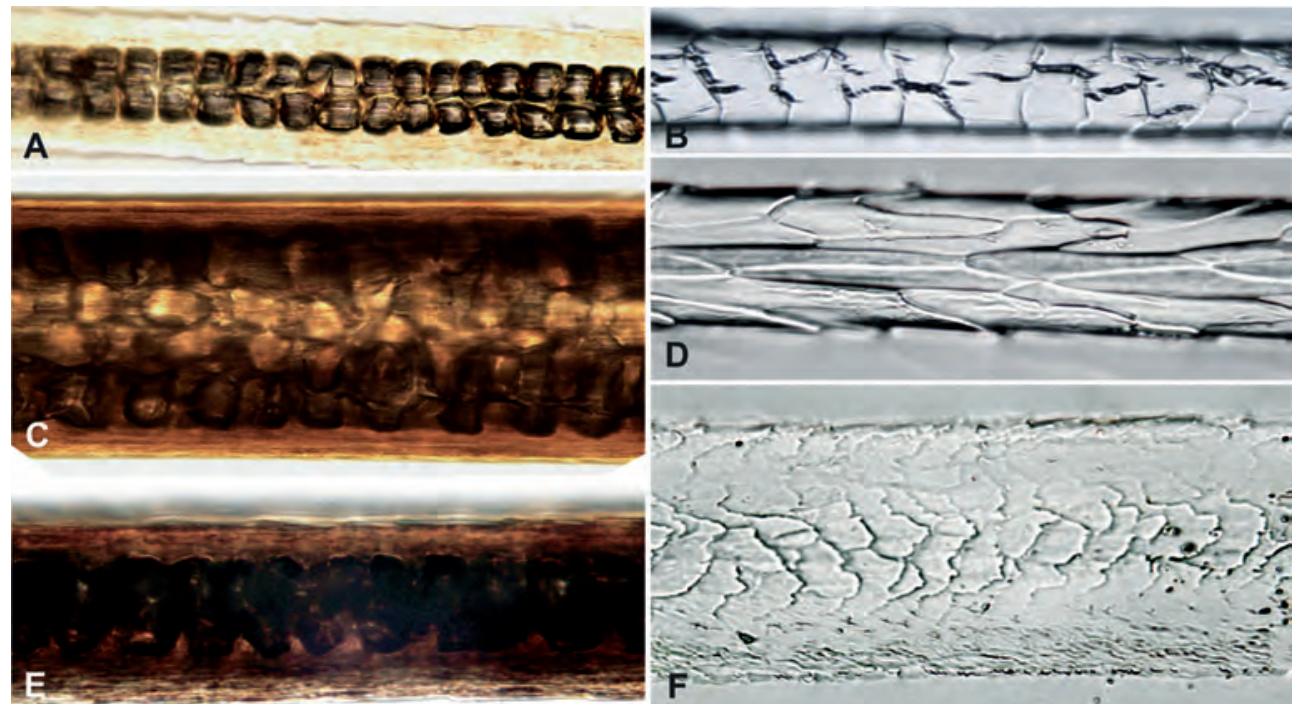

Figura 4: Patrones medulares y cuticulares de Lagidium viscacia (MUSA 8625, 4966). Porción proximal: Médula escaleriforme multiseriada (2 filas) (A) y Cutícula pétalo diamante "tipo B" (B). Escudo: Médula de forma reticular (C). Asta: Cutícula pétalo diamante "tipo D" (D). Porción distal: Médula en deformación (E) y Escamas ondeadas transversales $y$ crenadas (F).
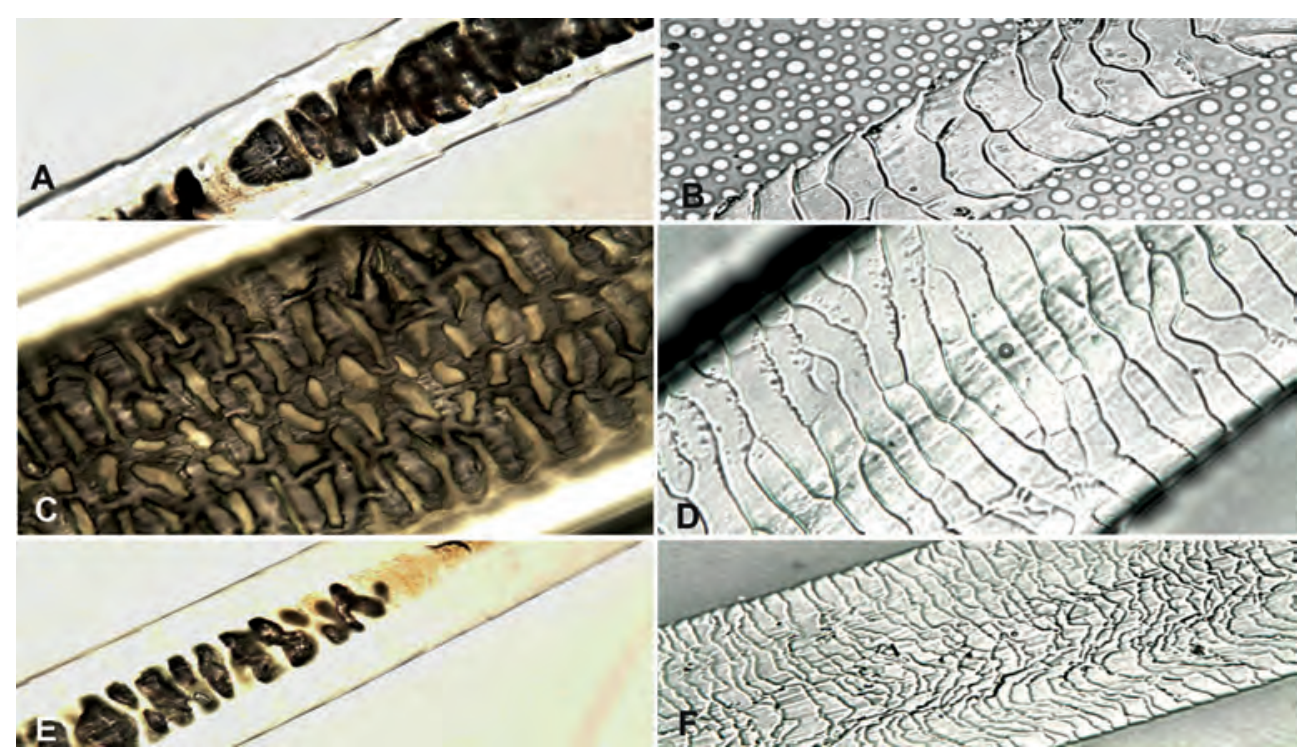

Figura 5: Patrones medulares y cuticulares Cavia spp. Fotos de la izquierda Cavia tschudii (MUSA 087) y fotos de la derecha $C$. porcellus (MUSA 5842). Porción proximal: Médula uniseriada tipo literoide(A) y Cutícula pétalo diamante "tipo A" (B). Escudo: Médula de forma reticular (C). Asta: Cutícula ondeada transversal y lisa (D). Porción distal: Médula en deformación (E) y Escamas ondeadas transversales y crenadas (F).
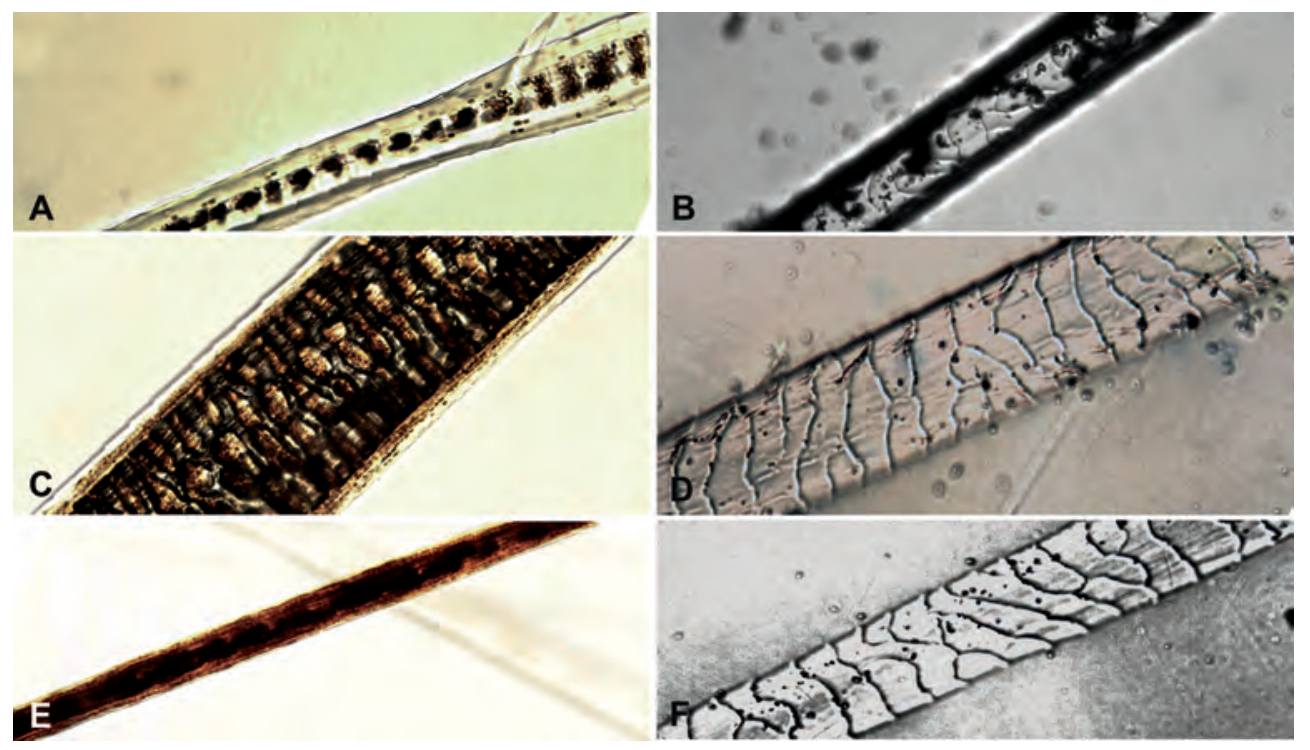

Figura 6: Patrones medulares y cuticulares de Abrocoma cinerea (MUSA 5104, 8625). Porción proximal: Médula escaleriforme uniseriada (A) y Cutícula pétalo diamante "tipo A" (B). Escudo: Médula de forma listrada (C). Asta: Cutícula ondeada oblicua simple y crenada (D). Porción distal: Médula en deformación (E) y Escamas ondeadas oblicuas simples y lisas (F). 


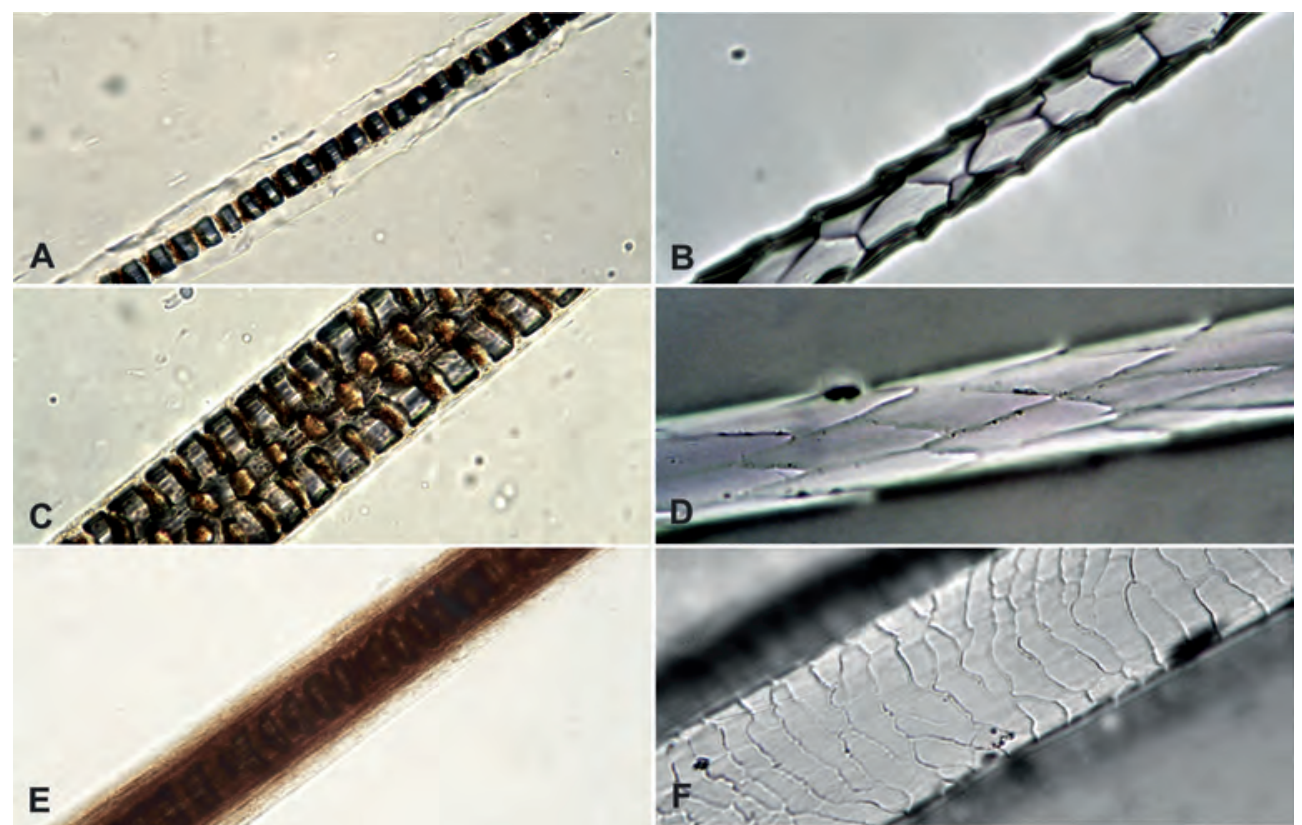

Figura 7: Patrones medulares y cuticulares en Cricetidae. Porción proximal: Médula escaleriforme uniseriada (Calomys chinchilico, MUSA 1568) (A) y Cutícula pétalo diamante "tipo A" (Aegialomys ica, MUSA 860) (B). Escudo: Médula de forma alveolar (Phyllotis limatus, MUSA 1258) (C). Asta: Cutícula foliácea oblicua simple y lisa (Abrothrix andinus, MUSA $1157)$ (D). Porción distal: Médula en deformación (Akodon subfuscus, MUSA 1279) (E) y Escamas ondeadas transversales y crenadas (Auliscomys sublimis, MUSA 1138) (F).

titativas entre las especies de dicha familia (Abrothrix andinus, A. jelskii, Akodon subfuscus, A. albiventer, Auliscomys pictus, A. sublimis, A. boliviensis, Calomys achaku, C. chinchilico, C. miurus, C. lepidus, Chinchillula sahamae, Necromys amoenus, Neotomys ebriosus, Oligoryzomys andinus, O. arenalis, Aegialomys ica, Phyllotis amicus, P. magister, P. limatus y P. xanthopygus).

Muridae.- Los representantes de esta familia se caracterizaron por presentar un patrón medular anastomosado reticular ( $R$. rattus) y alveolar $(R$. norvegicus y M. musculus) con bordes celulares íntegros (Fig. 8C, G y E). Sin embargo, $R$. rattus tiene patrón cuticular pétalo diamante "tipo C" (Fig. 8D), M. musculus tiene un patrón cuticular pétalo diamante "tipo B" (Fig. 8F), y R. norvegicus tiene un patrón cuticular foliáceo estrecho oblicuo y simple, en los tres casos los bordes de las escamas son continuos (Fig. 8H).

Respecto a los datos cuantitativos, el rango de LP en los representantes del orden Didelphimorphia fue de 9 a $13 \mathrm{~mm}$; mientras que en el orden Rodentia fue de 5 a 42 mm. Las medidas de los dos didélfidos son muy similares, lo mismo se observa entre los cávidos, mientras que en los cricétidos y múridos las medidas tienen variaciones (Tabla 4).

\section{Discusión}

De las cuatro zonas usadas en este estudio, las porciones proximales son muy parecidas entre sí; mientras que, las porciones distales son casi invariables entre todas las especies observadas. A nivel cuticular, el patrón comúnmente observado en la porción proximal fue de forma pétalo diamante esto concuerda con lo observado por Cavia et al. (2008). Por otro lado, la porción distal se deforma hasta desaparecer, esto fue descrito por Fernández y Rossi (1998) en su trabajo con roedores de Brasil.

Abreu et al. (2011) y Gatto et al. (2016) describieron los patrones medulares de los didélfidos Caluromys la- natus, Gracilinanus agilis, G. microtarsus, Marmosa paraguayana y Monodelphis cf. dimidiata, quienes reportaron el mismo patrón medular uniseriado escaleriforme reportado aquí para Thylamys; sin embargo, ninguno registró el patrón cuticular foliáceo estrecho observado para Thylamys pallidior y Thylamys sp.

En los cricétidos, todas las especies analizadas presentaron un patrón cuticular tipo foliáceo estrecho y una médula multiseriada tipo alveolar, concordando con Martin et al. (2009) y Silveira et al. (2013). Ambos autores describieron un patrón cuticular foliáceo ovalado, alargado u ondeado con la médula multiseriada alveolar o listrada en Akodon spp., Oligoryzomys nigripes, Necromys lasiurus y Calomys tener. Otros autores observaron patrones parecidos a los mencionados, pero los denominaron de otra manera: Fernández y Rossi (1998) mencionan patrones cuticulares de tipo lanceolado, mosaico, ondulado y pectinado, y un patrón medular bi, tri o multiseriado en $C$. venustus, $C$. musculinus, $C$. laucha, $C$. callosus, A. molinae y Phyllotis darwini; mientras que Gatto et al. (2016) describieron un patrón cuticular foliáceo estrecho y una médula listrada en $A$. montensis y $O$. nigripes. Esta variación en los patrones observados podría deberse a tres situaciones; 1) a pesar de que los especímenes son del mismo género, son especies diferentes de regiones geográficas diferentes; 2) las zonas del pelo observadas probablemente no sean las mismas; y 3) la variedad de bibliografía utilizada para la descripción, dado que a lo largo de los años cada autor ha nombrado y descrito los patrones observados según su propio criterio. A la fecha Quadros y Monteiro-Filho (2006b) han propuesto una clasificación y nomenclatura, tratando de sintetizar la información de los diversos tipos de patrones cuticulares y medulares, no obstante, aún hay algunos patrones que no han sido considerados en ese resumen.

En la familia Muridae se encontró que $R$. norvegicus y Mus musculus tienen médula multiseriada tipo alveolar 


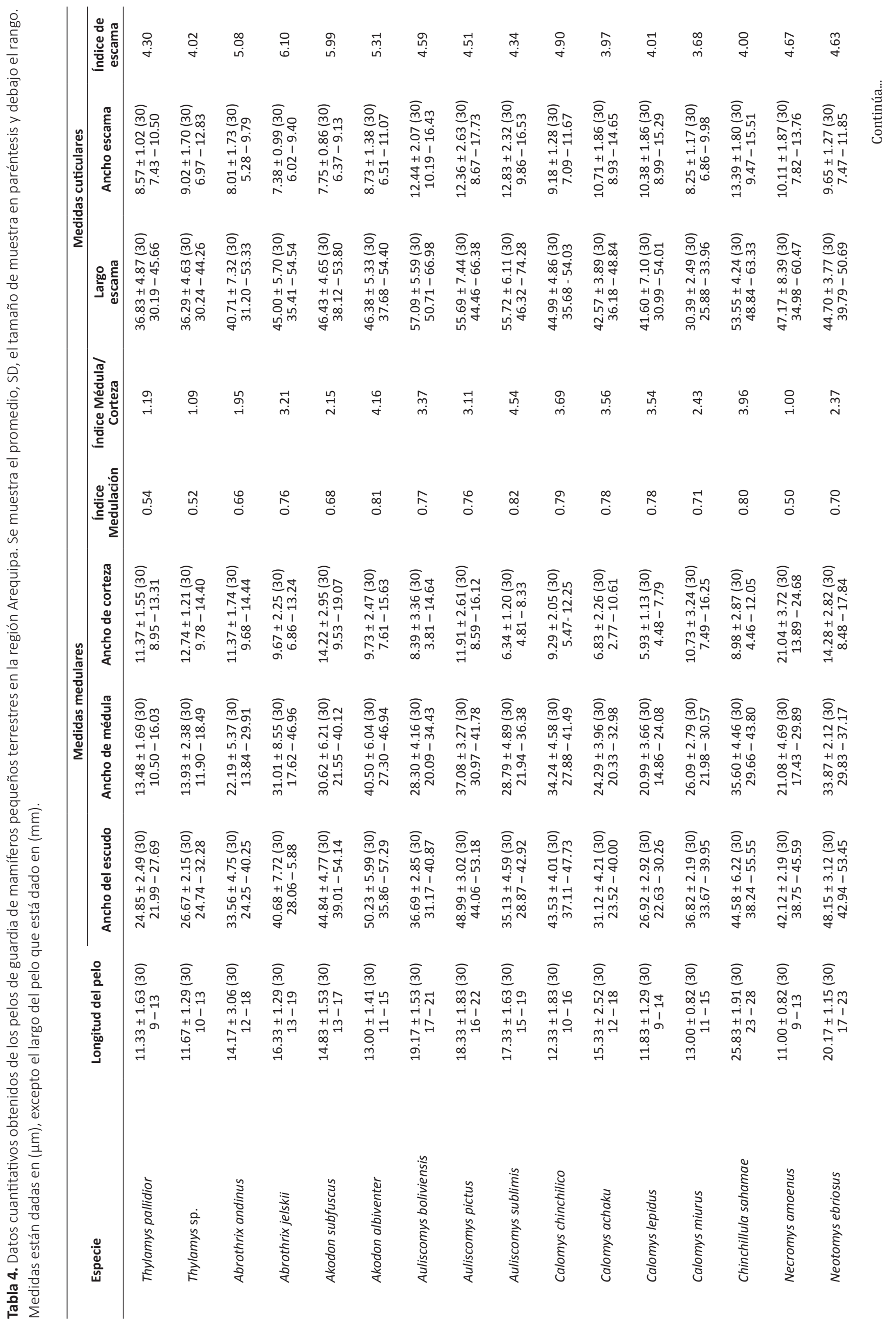




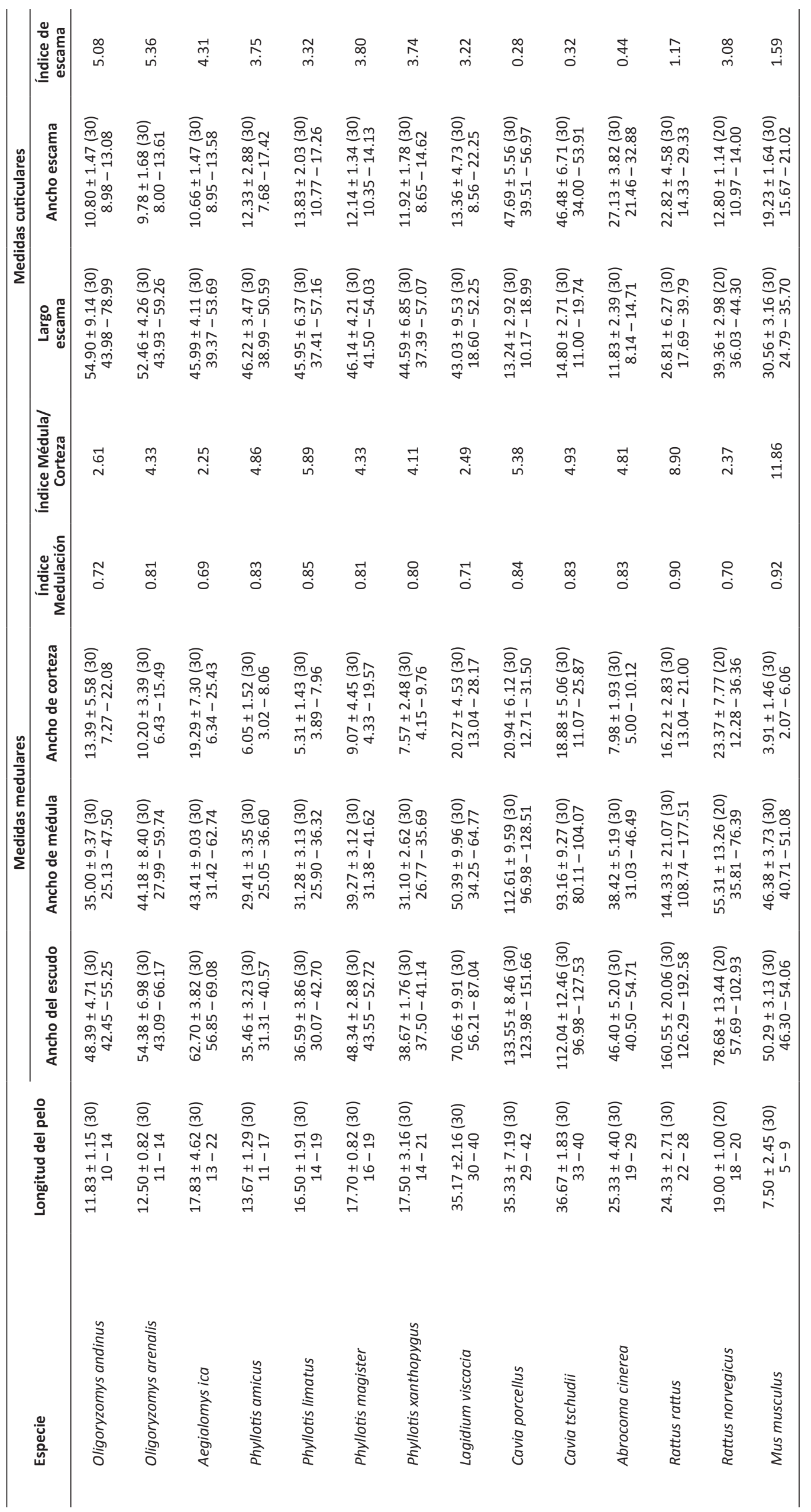



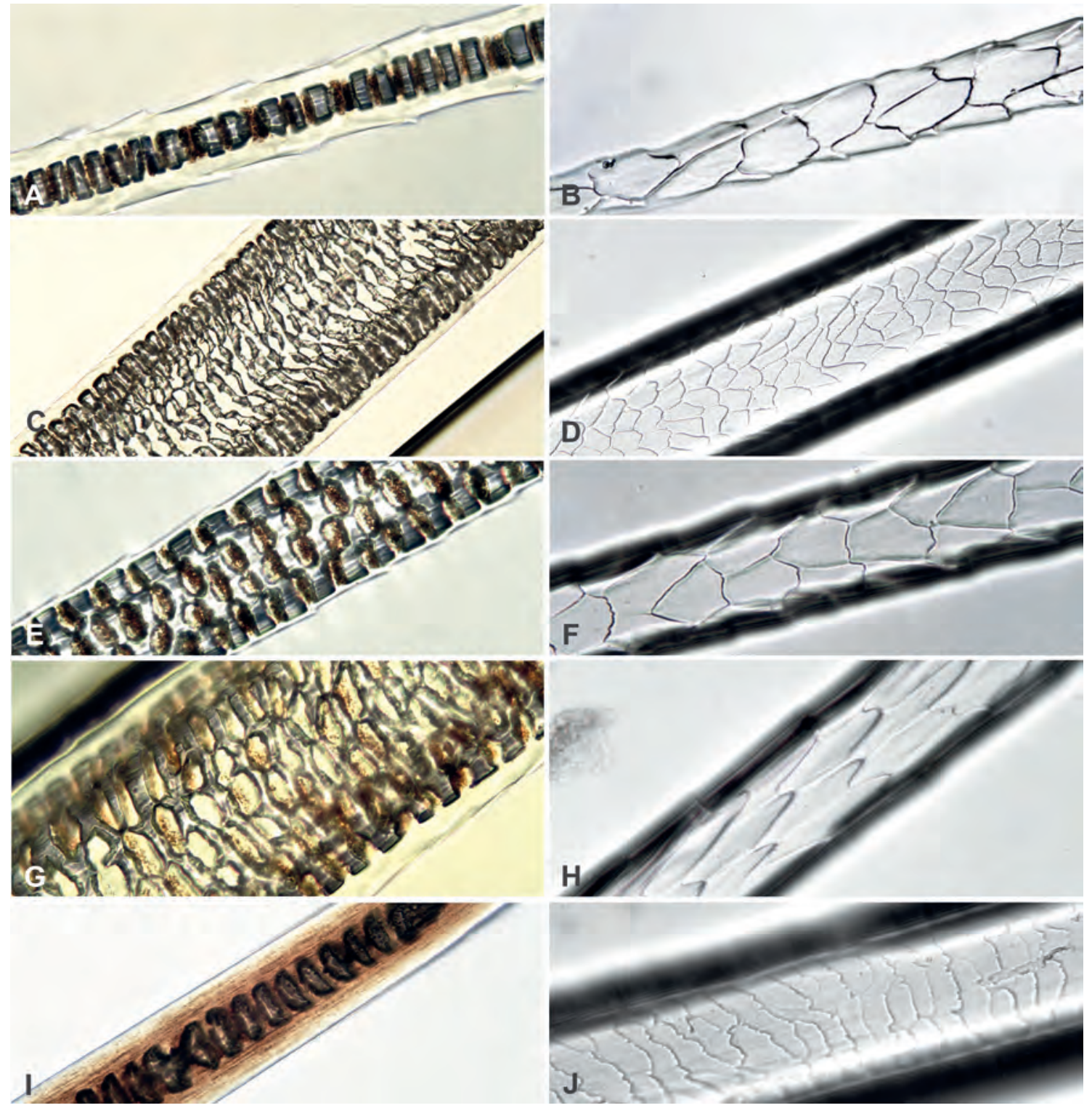

Figura 8: Patrones medulares y cuticulares en Muridae. Porción proximal: Médula escaleriforme uniseriada (Rattus rattus, MUSA 268) (A) y Cutícula pétalo diamante "tipo A" (Rattus norvegicus, MUSA 463) (B). Escudo: Médula de forma reticular ( $R$. rattus) (C), médula de forma alveolar (Mus musculus) (E) y (R. norvegicus) (G). Asta: Cutícula pétalo diamante "tipo C" (R. rattus) (D), Cutícula pétalo diamante "tipo B" (M. musculus, MUSA 584) (F) y Cutícula foliácea estrecha oblicua simple (R. norvegicus) (H). Porción distal: Médula escaleriforme en deformación (R. rattus) (I) y Escamas ondeadas transversales y crenadas ( $R$. norvegicus) (J).

y Rattus rattus un patrón medular reticular. $R$. rattus y M. musculus presentaron una cutícula pétalo diamante "tipo C y B" respectivamente, mientras que $R$. norvegicus presentó una cutícula tipo foliácea, concordando con Teerink (1991), Cavia et al. (2008), Pech-Canché et al. (2009) y Niroshini y Meegaskumbura (2015). En cambio, Debelica y Thies (2009) mencionan que $R$. rattus, $R$. norvegicus y $M$. musculus tienen una cutícula imbricada acuminada, con forma de $\mathrm{W}$ o tipo mosaico suave. Otros autores mencionan que $M$. musculus tiene médula con intrusiones corticales, escaleriforme, fragmentada tipo C o compuesta aplanada (Ibarra \& Sánchez-Cordero 2004; Pech-Canché et al. 2009; Debelica \& Thies 2009) y una cutícula tipo mosaico u ondeada irregular (Albayrak \&
Coban 1997; Ibarra \& Sánchez-Cordero 2004). Adicional a lo mencionado en los cricétidos, la variación en los patrones encontrados puede deberse a que la metodología utilizada para observar la médula del pelo fue variable, en este trabajo se decoloro el pelo en todos los casos; sin embargo, otros trabajos sólo decoloraron el pelo cuando era muy pigmentado, esta diferencia pudo ocasionar que la médula muestre un patrón diferente.

En cuanto a los chinchílidos y abrocómidos, aquí se presentan las primeras descripciones de los patrones cuticulares y medulares para Lagidium viscacia y Abrocoma cinerea.

En la familia Caviidae, Cavia tschudii y C. porcellus presentaron un patrón cuticular pavimentado ondeado 
transversal, el cual aparentemente también se presenta en Cavia aperea (Cavia et al. 2008), pero fue descrito como un patrón tipo mosaico. El patrón medular de $\mathrm{Ca}$ via tschudii y C. porcellus es multiseriado reticular; pero para $C$. aperea no se cuenta con esta información.

Algunos estudios utilizan los datos cuantitativos como caracteres diagnósticos para diferenciar entre especies de marsupiales y roedores (Cavia et al. 2008; Debelica \& Thies 2009); sin embargo, los datos cuantitativos obtenidos en este trabajo muestran muy poca variación entre especies de la misma familia, lo cual podría ocasionar un error en la correcta determinación de las especies. Nuestros datos apoyan las conclusiones de Monroy et al. (2005) quienes indicaron que las variables cuantitativas presentaron variación intraespecífica, por lo que no es recomendable usarlo como carácter principal de identificación; no obstante, a nivel de familias los datos numéricos obtenidos pueden ayudar a diferenciar entre las familias Cricetidae, Caviidae, Abrocomidae, Chinchillidae y Muridae.

En este trabajo presentamos por primera vez, los patrones cuticulares y medulares para los roedores, Cavia tschudii, Abrothrix andinus, A. jelskii, Akodon subfuscus, A. albiventer, Auliscomys pictus, A. sublimis, A. boliviensis, Calomys achaku, C. chinchilico, C. miurus, C. lepidus, Chinchillula sahamae, Necromys amoenus, Neotomys ebriosus, Oligoryzomys andinus, O. arenalis, Aegialomys ica, Phyllotis amicus, P. magister, P. limatus y P. xanthopygus.

Estos resultados se esperan sean útiles en diversos estudios ecológicos, como estudios en dieta de carnívoros donde los pelos son restos frecuentes (Chattha et al. 2011, Anwar et al. 2012, Canel et al. 2016), en la estimación de la distribución de mamíferos silvestres (Fasola et al. 2005, Schwingel \& Norment 2010), como un bioindicador no invasivo de contaminación por metales pesados en mamíferos (Obrusnik \& Paukert 1984, Gerstenberger et al. 2006), o incluso en estudios forénsicos como evidencia en investigaciones criminales, determinando la ubicación y circunstancias de un delito, así como la implicancia de los sospechosos en el delito (Robertson 1999).

\section{Literatura citada}

Abreu M.S.L., A.U. Christoff \& E.M. Vieira. 2011. Identificação de marsupiais do Rio Grande do Sul através da microestrutura dos pelos-guarda. Biota Neotropica 11(3):391400. http://www.biotaneotropica.org.br/v11n3/en/ abstract?identification-key +bn03111032011

Albayrak I. \& N. Coban. 1997. Hair morphology of some mammalian species in Turkey. Communications de la Faculte des Sciences de l'Universite d'Ankara. Series C. 15:21-39. https://doi.org/10.1501/Commuc_0000000097

Anwar M.B., M.S. Nadeem, M.A. Beg, A.R. Kayani \& G. Muhammad. 2012. A photographic key for the identification of mammalian hairs of prey species in snow leopard (Panthera uncia) habitats of Gilgit-Baltistan Province of Pakistan. Pakistan Journal of Zoology 44(3):737743.
Arita H. \& M. Aranda. 1987. Técnicas para el estudio y clasificación de los pelos. Instituto Nacional de Investigaciones sobre Recursos Bióticos, Xalapa, Veracruz. Cuadernos de Divulgación Inireb $\mathrm{N}^{\circ} 32$.

Benedict F.A. 1957. Hair structure as a generic character in bats. University of California. Publications in Zoology Berkeley 59:285-548.

Brunner H. \& B.J. Coman. 1974. The identification of mammalian hair. Melbourne, Inkata Press.

Canel D., N.P. Scioscia, G.M. Denegri \& M. Kittlein. 2016. Dieta del zorro gris pampeano (Lycalopex gymnocercus) en la provincia de Buenos Aires. Mastozoología Neotropical 23(2):359-370. http:// www.scielo.org.ar /scielo.php?script=sci_ arttext\&pid=S0327-93832016000200012\&lng=es.

Cavia R. et al. 2008. Hair structure of small rodents from central Argentina: A tool for species identification. Mammalia 72(1): 35-43. https://doi.org/10.1515/ MAMM.2008.009

Chattha S.A., K.M. Anjum, M. Altaf \& M.Z. Yousaf. 2011. Hair mounting technique: helpful in conservation of carnivores. FUUAST Journal of Biology 1(2): 53.

Day M.G. 1966. Identification of hair and feather remains in the gut and feaces of stoats and weasels. Journal of Zoology London 148:201-217. https://doi. org/10.1111/j.1469-7998.1966.tb02948.x

De Marinis A.M. \& P. Agnelli. 1993. Guide to the microscope analysis of Italian mammals' hairs: Insectivora, Rodentia and Lagomorpha. Italian Journal of Zoology 60(2):225-232.

Debelica A. \& M.L. Thies. 2009. Atlas and Key to the hair of terrestrial Texas mammals. Special Publications (No. 55). Museum of Texas Tech University. Natural Science Research Laboratory. Texas Tech University. 102 pp. https://doi.org/10.5962/bhl.title.142652

do Prado J.R. \& A.R. Percequillo. 2018. Systematic studies of the genus Aegialomys Weksler et al., 2006 (Rodentia: Cricetidae: Sigmodontinae): Geographic variation, species delimitation, and biogeography. J Mammal Evol 25: 71. https://doi.org/10.1007/s10914-016-9360-y

Fasola L., M. Bello \& M.L. Guichón. 2005. Uso de trampas de pelo y caracterización de los pelos de la ardilla de vientre rojo Callosciurus erythraeus. Mastozoología Neotropical 12(1):9-17.

Felix G.A., U. Piovezan, J. Quadros, R.S. Juliano, F.V. Alves \& M.C.S. Fioravanti. 2014. Thricology for identifying mammal species and breeds: its use in research and agriculture. Archivos de Zootecnia 63(R):107-116.

Fernández G. \& S. Rossi. 1998. Medullar type and cuticular scale patterns of hairs of rodents and small marsupials from the Monte Scrubland (San Luis province, Argentina). Mastozoología Neotropical 5(2):109-116.

Gatto-Almeida F., J.S. Pontes, I.J. Sbalqueiro, I. Hass, L.M. Tiepolo \& J. Quadros. 2016. Diversidade, biogeografia, caracterização cariotípica e tricológica dos pequenos mamíferos não voadores do Parque Estadual Rio da Onça, Litoral Sul do Paraná. Papéis Avulsos de Zoologia 56(7):69-96. http://dx.doi.org/10.11606/00311049.2016.56.07

Gerstenberger S.L., C.L. Cross, D.D. Divine, M.L. Gulmatico \& A.M. Rothweiler. 2006. Assessment of mercury concentrations in small mammals collected near Las Vegas, Nevada, USA. Environmental toxicology 21(6):583-589. https://doi.org/10.1002/tox.20221 
Gómez J.J. \& M.H. Cassini. 2010. Uso de pelos de guardia para la identificación de mustélidos costeros en la Patagonia. Revista de Biología Marina y Oceanografía 45(2):359-363. https://doi.org/10.4067/S071819572010000200021

Gurini L.B. 1985. Valor diagnóstico del pelaje y su aplicación al estudio al estudio de las interacciones tróficas, con referencia a especies del Delta Bonaerense. 179 pp. Tesis de Doctorado, Universidad Nacional de La Plata, La Plata, Argentina.

Hausman L.A. 1920. Structural characteristics of the hair of mammals. The American Naturalist 64(635):496-523. https://doi.org/10.1086/279782

Ibarra I.I.B. \& V. Sánchez-Cordero. 2004. Catálogo de pelos de guardia dorsal en mamíferos del estado de Oaxaca, México. Anales del Instituto de Biología-Serie Zoología 75(2):383-437.

Juárez-Sánchez A.D., C.G. Estrada, M. Bustamante, Y. Quintana \& J.E. López. 2007. Guía ilustrada de pelos para la identificación de mamíferos medianos y mayores de Guatemala. Dirección General de Investigación, Universidad de San Carlos de Guatemala, 28. 107 pp.

Keller A. 1980. Détermination des mammìferes de la Suisse par leur pelage: II. Diagnose des Families, III Lagomorpha et Rodentia. Revue Suisse de Zoologie, Geneve 87:781796. https://doi.org/10.5962/bhl.part.85550

Kshirsagar S.V., B. Singh \& S.P. Fulari. 2009. Comparative study of human and animal hair in relation with diameter and medullary index. Indian Journal of Forensic Medicine and Pathology 2(3):105-108.

Kumar M., Y.S. Priya, V. Mathur, H. Kumar \& V. Elangovan. 2014. Scanning electron microscopy study of guard hair of three Indian pteropodid bats. Mammalia 78(4):533537. https://doi.org/10.1515/mammalia-2013-0103

Martin R.E., R.H. Pine \& A.F. Deblase. 2001. A manual of mammalogy: with keys to families of the world. Third Edition. Waveland Press, Inc.

Martin P.S., C. Gheler-Costa \& L.M. Verdade. 2009. Microestruturas de pêlos de pequenos mamíferos não-voadores: chave para identificação de espécies de agroecossistemas do estado de São Paulo, Brasil. Biota Neotropica 9(1):233. https://doi.org/10.1590/S167606032009000100022

Monroy-Vilchis O. et al. 2005. Variación intraespecífica e individual de los pelos de mamíferos del estado de México: implicaciones en la identificación interespecífica. CIENCIA ergo sum 12(3):264-270.

Niroshini D.M.C. \& S. Meegaskumbura. 2015. Identification of Sri Lankan muroid rodents using hair anatomy. Ceylon Journal of Science (Biological Sciences) 43(2). https://doi.org/10.4038/cjsbs.v43i2.7322

Obrusnik I. \& J. Paukert. 1984. Indication of environmental pollution by means of INAA of the hair of some free-living mammals. Journal of radioanalytical and nuclear chemistry 83(2):397-406. https://doi.org/10.1007/ BF02037154

Palma R.E. et al. 2014. Filogenética molecular de zarigüeyas: nuevos hallazgos sobre la filogenia de Thylamys (Didelphimorphia, Didelphidae). Zoologica Scripta 43(3):217-234. https://doi.org/10.1111/zsc.12051

Patton J.L., U.F. Pardi-as \& G. D'elía. (Eds.). 2015. Mammals of south America, Rodents (Vol. 2). University of Chicago Press. https://doi.org/10.7208/chicago/9780226169606.001.0001
Pech-Canché J.M., J.E. Sosa-Escalante \& M.E.K. Cruz. 2009. Guía para la identificación de pelos de guardia de mamíferos no voladores del Estado de Yucatán, México. Revista Mexicana de Mastozoología (Nueva época) 13(1):733.

Perrin M.R. \& B.S. Campbell. 1980. Key to the mammals of the Andries Vosloo Kudu Reserve (Eastern Cape), based on their hair morphology, for use in predator scat analysis. South African Journal of Wildlife Research, Stellenbosch 10:1-14.

Quadros J. 2002. Identificação microscopic de pelos de mamíferos brasileiros e sua aplicação no estudo da dieta de carnívoros. Tese inédita Universidade Federal do Paraná, Curitiba, Paraná, Brasil.

Quadros J. \& E.L. de A. Monteiro-Filho. 2006a. Revisão conceitual, padrões microestrutuais e proposta nomenclatória para os pêlos-guarda de mamíferos brasileiros. Revista Brasileira de Zoología 23(1):279-292. https:// doi.org/10.1590/S0101-81752006000100023

Quadros J. \& E.L. de A. Monteiro-Filho. 2006b. Coleta e preparação de pêlos de mamíferos para identificação em microscopia óptica. Revista Brasileira de Zoologia 23(1):274-278. https://doi.org/10.1590/S010181752006000100022

Robertson J. 1999. Forensic examination of hair. Taylor y Francis Inc. Philadelphia. https://doi. org/10.1201/9780203483527

Sarkar P.S. \& J.K. De. 2013. Tricho-taxonomic study of dorsal guard hairs of Indian species of rodents belonging to Subfamily-Sciurinae (Sciuridae: Rodentia: Mammalia). Biological Forum - An International Journal 5(1):1-10.

Schwingel H. \& C. Norment. 2010. Use of hair tubes to detect small-mammal winter activity in a northern forest habitat. Northeastern Naturalist 17(4): 531-540. https://doi.org/10.1656/045.017.0402

Silveira F., I.J. Sbalqueiro \& E.L. de A. Monteiro-Filho. 2013. Identification of the brazilian species of Akodon (Rodentia: Cricetidae: Sigmodontinae) through the microstructure of the hair. Biota Neotropica 13(1):339-345. 0611. https://doi.org/10.1590/ S1676-06032013000100033

Teerink B.J. 1991. Hair of west european mammals: atlas and identification. Cambridge, Cambridge University Press.

Vázquez D., P. Perovic \& A. de Olsen. 2000. Patrones cuticulares y medulares de pelos de mamíferos del noroeste argentino (Carnivora y Artiodactyla). Mastozoología Neotropical 7(2):131-147.

Zeballos H., V. Pacheco \& L. Baraybar. 2001. Diversidad y conservación de los mamíferos de Arequipa, Perú. Revista Peruana de Biología 8(2):94-104. http://dx.doi. org/10.15381/rpb.v8i2.6564

Zeballos H., R.E. Palma, P.A. Marquet \& G. Ceballos. 2014. Phylogenetic relationships of Calomys sorellus complex (Rodentia: Cricetidae), with the description of two new species. Revista Mexicana de Mastozoología (Nueva época) 4(1):1-23. https://doi.org/10.15450/ RMM141001 
Agradecimientos

A los investigadores asociados y voluntarios de la Colección Científica del Museo de Historia Natural (MUSA), en especial a los miembros del área de mastozoología, Dr. Horacio Zeballos, Alexander Pari, Kateryn Pino, Jean Paul Ludeña, Gabriel Llerena y Alaín Escobar, por haber ayudado a formar una gran colección científica de mamíferos. Tambien al apoyo en la gestión de CIENCIACTIVA y CONCYTEC

Rol de los autores:

YKM y CEM prepararon el primer borrador del manuscrito. EL asesoro la tesis y reviso la versión final del manuscrito.
Conflicto de intereses:

Los autores no incurren en conflictos de intereses.

Fuentes de financiamiento:

Universidad Nacional de San Agustín de Arequipa (UNSA), mediante Contrato de subvención $\mathrm{N}^{\circ}$ 14-2016-UNSA.

Aspectos éticos / legales:

Este trabajo no incurrio en ningun problema ético ni legal.

Apéndice I. Especímenes examinados en este estudio, con sus respectivas localidades.

1. Thylamys pallidior (3) -Arequipa: Condesuyos, Ispacas (MUSA 14400); Castilla, Huanacaure (MUSA 2764); Puno: Chucuito, Hda. Ventilla (MUSA 4460).

2. Thylamys sp. (3) -Arequipa: Caylloma, Ichupampa (MUSA 8299); La Unión, Uchucllayco (MUSA 11553); Caravelí, Lomas de Atiquipa (MUSA 7353).

3. Abrothrix andinus (3) -Arequipa: Caylloma, Tocra (MUSA 931); Arequipa, Salinas Huito (MUSA 1154, 1157).

4. Abrothrix jelskii (3) -Arequipa: Caravelí, Chaquicocha (MUSA 4973); Junín: Jauja, CanchaylloPuya (MUSA 5327, Laguna Tranca grande (MUSA 16925).

5. Akodon subfuscus (3) -Arequipa: Caylloma, Linde (MUSA 8266); Castilla, Huanacaure (MUSA 2768); Arequipa, Cayma (MUSA 1279).

6. Akodon albiventer (3) -Tacna: Palca, Alto Perú (MUSA 12457); Tarata (MUSA 3418); Puno, Chucuito, Hda. Ventilla (MUSA 4560).

7. Auliscomys boliviensis (3) -Arequipa: Pati, R. N. Salinas y Aguada Blanca (MUSA 1122, 1128); Caylloma, Puquiolacca (MUSA 1118).

8. Auliscomys pictus (3) -Arequipa: Caylloma, Puquiolacca (MUSA 927, 1135); El Rayo (MUSA 7201).

9. Auliscomys sublimis (3) -Arequipa: Caylloma, $\mathrm{Pu}$ quiolacca (MUSA 1140); Imata (MUSA 1138); Chajhui, Hda. Quilcapata (MUSA 082).

10. Calomys chinchilico (3) -Arequipa: Caravelí, Lomas de Taymara, Atiquipa (MUSA 1568-1570).
11. Calomys achaku (3) -Arequipa: Caylloma, Pillone, Hda. Jjocca (MUSA 075); Chaco, Río Colca (MUSA 272); Chivay, Río Colca (MUSA 280).

12. Calomys lepidus (3) -Arequipa: Pampa Cañahuas, R. N. Salinas y Aguada Blanca (MUSA 7127, 7130); San Juan de Tarucani (MUSA 7137).

13. Calomys miurus (3) -Junín: Jauja, Jauja (MUSA 4673); Canchayllo-Puya, R. P. Nor Yauyos Cochas (MUSA 5340); Lima: Vilca-Huancaya, bosque Karka, R. P. Nor Yauyos Cochas (MUSA 5355).

14. Chinchillula sahamae (3) -Arequipa: Caylloma, El Rayo (MUSA 7222); Tocra (MUSA 5132, 1108).

15. Necromys amoenus (3) -Puno: Chucuito, Challacollo (MUSA 12407); Hda. Ventilla, entre Pichupichuni y Huacullani (MUSA 4453); Cusco: Chumbivilcas, Laguna Japuccocha (MUSA 13594).

16. Neotomys ebriosus (3) -Arequipa: Castilla, Rio Orcopampa margen izqda. (MUSA 14015, 14016); Cayma, Qda. Písac (MUSA 1149).

17. Oligoryzomys andinus (3) -Arequipa: Castilla, Acho, Ayo (MUSA 2672, 2709); Chiguata (MUSA 060).

18. Oligoryzomys arenalis (3) -Arequipa: Islay, Lomas de Cocachacra (MUSA 13528); Mejía, Lomas de Challascapa (MUSA 689); Caravelí, Lomas de Atiquipa (MUSA 7344).

19. Aegialomys ica (3) -Arequipa: Caravelí, Lomas de Atiquipa (MUSA 855, 860, 7334).

20. Phyllotis amicus (3) -Arequipa: Caravelí, Lomas de Taymara (MUSA 5656, 5657); La Cueva, Chaquicocha (MUSA 4968).

21. Phyllotis limatus (3) -Arequipa: Yarabamba (MUSA 322, 326); Cayma (MUSA 1258). 
22. Phyllotis magister (3) -Arequipa: Caravelí, Chaquicocha (MUSA 4969); Caylloma, Ichupampa (MUSA 8297); R. N. Salinas y Aguada Blanca, Cayma (MUSA 8404).

23. Phyllotis xanthopygus (3) -Arequipa: Caylloma, Tocra, Yanque (MUSA 5137, 5142); El Rayo, Yanque (MUSA 7226).

24. Lagidium viscacia (3) -Arequipa: Caylloma, Vizcachani (MUSA 8625); Tocra Pampa (MUSA 4182); Caravelí, La Cueva, Chaquicocha (MUSA 4966).

25. Cavia porcellus (3) -Arequipa: Castilla, Pedregal (MUSA 215); El Cural (MUSA 099); Arequipa (MUSA 5842).
26. Cavia tschudii (3) -Arequipa: Yarabamba (MUSA 087); Tiabaya (MUSA 089); Cerro Colorado, El Cural (MUSA 098).

27. Abrocoma cinerea (3) -Arequipa: Cayma, Cabrerías (MUSA 7403); Qda. Písac (MUSA 1103); Caylloma, Tocra (MUSA 5104).

28. Rattus rattus (3) -Arequipa: Cerro Colorado, El Cural (MUSA 094); Cayma (MUSA 268); Tiabaya, Cerro San Ignacio (MUSA 055).

29. Rattus norvegicus (2) -Arequipa: Islay, Dean Valdivia (MUSA 463); Lima: Cañete, R. P. Nor Yauyos Cochas, Huantan (MUSA 5177).

30. Mus musculus (3) -Arequipa: Mollebaya (MUSA 196); Chiguata (MUSA 584); Islay, Mejía (MUSA 684). 